\title{
Localizing particles showering in a Spaghetti Calorimeter
}

D. Acosta ${ }^{a}$, S. Buontempo ${ }^{b}$, L. Calôba ${ }^{c}$, M. Caria ${ }^{\text {d }}$, R. DeSalvo ${ }^{f}$, A. Ereditato ${ }^{b}$, R. Ferrari ${ }^{\text {h }}$, G. Fumagalli ${ }^{\text {h }}$, G. Goggi ${ }^{\text {h }}$, W. Hao ${ }^{g, *}$, F.G. Hartjes ', A. Henriques ${ }^{f_{J}}$, L. Linssen ${ }^{f}$, M. Livan ${ }^{d}$, A. Maio ', L. Mapelli ${ }^{\text {e }}$, M.R. Mondardini ${ }^{k}$, B. Ong ${ }^{a}$, H.P. Paar ${ }^{\text {a }}$, F. Pastore ${ }^{\text {h }}$, M. Pereira ${ }^{j}$, L. Poggioli ${ }^{~}$, F. Riccardi ${ }^{b}$, C.V. Scheel ${ }^{\text {f,k }}$, J. Schmitz ${ }^{\text {i }}$, J.M. Seixas ${ }^{\text {c,g }}$, A. Simon ${ }^{\mathrm{m}}$, M. Sivertz ${ }^{a}$, P. Sonderegger ${ }^{\mathrm{e}}$, M.N. Souza ${ }^{c}$, Z.D. Thome $^{c}$, V. Vercesi ${ }^{\mathrm{h}}$, Y. Wang ${ }^{\mathrm{g}, *}$, $\mathrm{R}$. Wigmans ${ }^{\mathrm{f}, \mathrm{i}}$ and $\mathrm{C} . \mathrm{Xu}{ }^{\mathrm{g}, *}$

${ }^{a}$ Unversity of Calfornia, San Diego, USA

${ }^{b}$ Università di Napolt and INFN Sez. Napoli, Italy

'COPPE/EE/UFRJ, Rio de Janetro, Brazll

${ }^{d}$ Universıtò di Cagliarı and INFN Sez. Caghari, Italy

${ }^{e}$ CERN, Geneva, Switzerland

${ }^{f}$ CERN/LAA project, Geneva, Switzerland

${ }^{8}$ CERN (Worldlab fellowship), Geneva, Switzerland

${ }^{h}$ Università di Pavia and INFN Sez. Pavia, Italy

'NIKHEF-H, Amsterdam, The Netherlands

'LIP, Lisbon, Portugal

${ }^{k}$ Cornell University, Ithaca, USA

${ }^{\prime}$ LPNHE, Unversité Paris VI and VII, Paris, France

${ }^{m}$ Universtät Heidelberg, Heidelberg, Germany

Received 23 January 1991

We report on the performance of a fine-grained 13-ton compensating lead/scintillating-fiber calorimeter, and in particular on its capability of localizing the particles that produce showers in it. The RMS position resolution was found to be $1.7 \mathrm{~mm}$ for electromagnetic showers and $5.1 \mathrm{~mm}$ for hadronic showers at $80 \mathrm{GeV}$, averaged over a tower with an effective radius of $39 \mathrm{~mm}$. Pion-pion separation through analysis of the energy deposit pattern was achieved in more than $95 \%$ of the cases for distances down to $8 \mathrm{~cm}$ at $80 \mathrm{GeV}$. Because of the good lateral position resolution, detailed information on the longitudinal shower development could be obtained, with the help of tracking information, when single particles entered the (longitudinally unsegmented) detector at a small angle with respect to the fiber axis. This information made it possible to eliminate the effects of light attenuation in the fibers on the hadronic energy resolution and allowed $\mathrm{e} / \pi$ separation at the $10^{-4}$ level.

\section{Introduction}

The new generation of multi-TeV high-luminosity pp colliders (LHC, SSC) requires detectors of unprecedented quality in order to allow for meaningful experiments. Calorimetry is among the most crucial elements of experiments at such machines, since it has to provide the first-level trigger information. It is the first filter for selecting the rare $\left(10^{-8}\right.$ level) interesting events under extreme background conditions. Moreover, the calorimeter information is the basis for studying the production of jets, electrons and missing energy, the most likely messengers of new physics.

\footnotetext{
* On leave of absence from IHEP Beijing, China.
}

The Spaghetti Calorimeter (or SPACAL) was developed, in the framework of the LAA project at CERN, with these goals in mind. It is a compensating lead/ scintillating-fiber calorimeter with monolythic towers, i.e. without longitudinal segmentation into an electromagnetic (e.m.) and an hadronic section.

Among its remarkable properties, we mention two that are particularly relevant for its performance in an LHC/SSC environment, namely the very high signal speed [1] and the hermeticity. The latter is achieved because the fibers that sample the showers are running (approximately) in the same direction as the incoming particles, so that all the readout is located at the rear detector end [2].

In a typical event at the $\mathrm{LHC} / \mathrm{SSC}$, more than 100 secondary particles will be produced. Moreover, multi- 
ple events per bunch crossing will frequently occur when the luminosity exceeds $10^{33} \mathrm{~cm}^{-2} \mathrm{~s}^{-1}$. Therefore, the spatial resolving power of the calorimeter is crucial for extracting the physics information, e.g. on jet and electron production and, in combination with other detectors, on multiple vertices.

In this paper, we report on the properties of the SPACAL calorimeter in this respect. In section 2, the detector and the experimental setup for the data taking are described. In section 3, the results on the position resolution for e.m. shower detection are given, and section 4 deals with the hadronic position resolution.

The particle-particle separation greatly benefits from the high density and hence from the short radiation and interaction length of the detector. Experimental results are described in section 5 . In section 6 , we demonstrate that the excellent position resolution provides a tool for improving the energy resolution and the electron identification capability of the detector. Conclusions are given in section 7 .

\section{Experimental setup}

\subsection{The detector}

The measurements were performed with a calorimeter consisting of 155 monolythic towers. Each tower contains 1141 plastic scintillating fibers ${ }^{\# 1}$ with a diameter of $1 \mathrm{~mm}$ and a length of $2.20 \mathrm{~m}$. These fibers form the active part of this sampling calorimeter. They are embedded in a lead matrix in such a way that each fiber is equidistant to its six neighbours (fig. 1a). The fiber spacing is $2.22 \mathrm{~mm}$ (centre-to-centre), such as to achieve a volume ratio lead : fiber of $4: 1$, needed in order to make the calorimeter compensating [3,4].

The fibers were grouped to form 155 towers. Each tower has an hexagonal cross section $(86 \mathrm{~mm}$ apex-toapex). The depth of the lead structure is $200 \mathrm{~cm}$. The fibers sticking out at the back end of the tower were bunched together in an hexagonal structure, machined and polished and coupled through an hexagonal light guide ( $79 \mathrm{~mm}$ long, $42 \mathrm{~mm}$ apex-to-apex) to a photomultiplier (PM) ${ }^{\# 2}$. The front end of a fiber was polished and made reflective by aluminium sputtering, such as to make the response more uniform as a function of depth. More detailed information about the structure of the calorimeter can be found in ref. [2]. The lateral cross section of the detector as a whole is shown in fig. $1 \mathrm{~b}$. The central tower is surrounded by seven concentric hexagonal rings; with the outer ring incomplete, the

\footnotetext{
\#1 SCSF-38, produced by Kyowa Gas, now Kuraray Co. Ltd, Tokyo, Japan.

\#2 Philips XP 2282, 8-stage.
}

a)
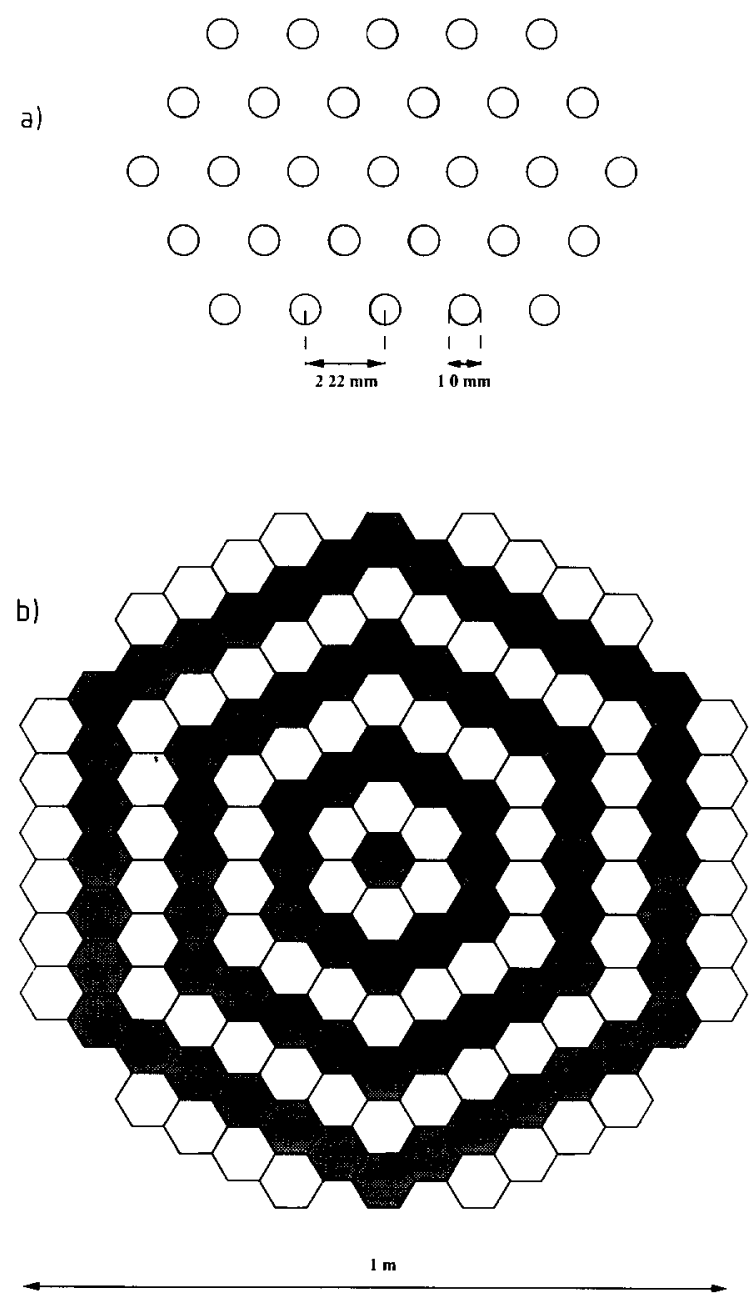

Fig. 1. Detail of the front face of the calorimeter (a) and the lateral structure of the detector as a whole (b).

detector has a roughly cylindrical shape with a diameter of $1 \mathrm{~m}$.

Because of the large fraction of high- $Z$ absorber material ( $80 \%$ in volume), the shower dimensions are relatively small in this detector. The effective radiation length $X_{0}$ amounts to $7.5 \mathrm{~mm}$, the effective Molière radius $R_{\mathrm{M}}$ to $25 \mathrm{~mm}$, the effective nuclear interaction length [5] $\lambda_{\mathrm{I}}$ to $21 \mathrm{~cm}$ and the average density to 9.3 $\mathrm{g} / \mathrm{cm}^{3}$. The sampling fraction for showers is only $2.3 \%$. The detector is, therefore, almost $10 \lambda_{I}$ deep and almost $5 \lambda_{\mathrm{I}}$ across, enough to contain $150 \mathrm{GeV} \pi^{-}$showers on average to better than $98 \%$, as we will show in a forthcoming paper.

The PM signals were handled as follows (see fig. 2). The anode signal was split into two equal parts by means of a passive splitter inside the base. One part went to the counting room where it was further fed into an active splitter, one output of which was sent un- 


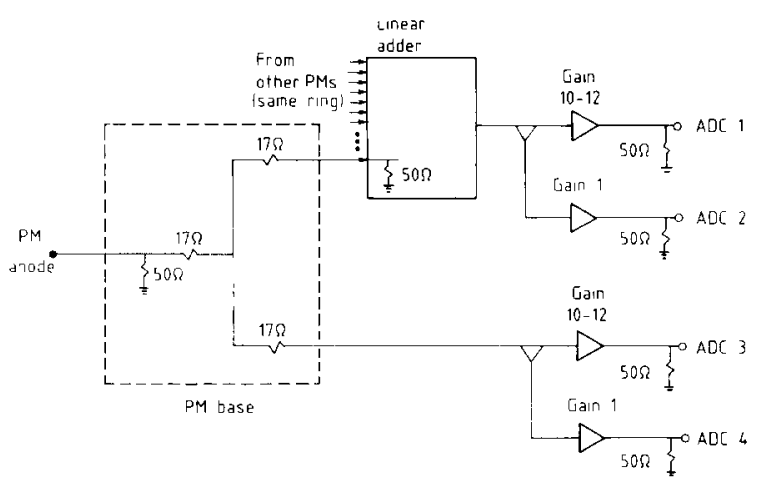

Fig. 2. The handling of the photomultiplier signals. See text for detalls.

changed into a 12-bit charge ADC (LeCroy 2280), the other output was amplified by a factor of $\sim 10$ before being fed into an ADC. The other half of the anode signal went into a linear adder, where it was combined with tower signals from the same hexagonal ring. The resulting ring sum signals were treated in the same way as the signals that were directly sent to the counting room.

The gain in the PM tube was set to $\sim 4 \mathrm{pC} / \mathrm{GeV}$ in the central detector region, gradually going up to -20 $\mathrm{pC} / \mathrm{GeV}$ in the outer rings, for the unamplified channels. The ADC gain was 4 counts $/ \mathrm{pC}$. This procedure was chosen to circumvent the insufficient dynamic range of our PM/ADC system and to be sensitive to small energy deposits far away from the shower axis.

Sparse data readout was enabled: signals smaller than 4 counts above the pedestal value were not recorded. This corresponds to a cutoff of $5 \mathrm{MeV}$ energy deposits in the amplified channels of the outer rings.

\subsection{The beam line}

The measurements were performed in the $\mathrm{H} 2$ beam line of the SPS at CERN. The detector was mounted on a platform that could move horizontally and vertically with respect to the beam line, with an accuracy of about $1 \mathrm{~mm}$. Moreover, the detector could be rotated around its vertical axis, so that the particles could be sent into the detector at a chosen angle $\theta_{z}$ (usually a few degrees) with respect to the fiber axis. The accuracy of the angular movement was better than $0.1 \mathrm{mrad}$.

Behind the detector, a smaller (20-tower) prototype of the same structure was installed. In practice, we used this detector to identify and remove muons from the data samples, and to measure longitudinal shower leakage. Upstream of the calorimeter, a trigger counter telescope was installed. It consisted of 5 scintillation counters ( $\mathrm{S} 1-\mathrm{S} 5)$ and 2 drift chambers with $x, y$ readout $(\mathrm{BC} 1, \mathrm{BC} 2)$. The layout is shown in fig. 3 .

Beams of electrons and negative pions of $5,10,20$, 40,80 and $150 \mathrm{GeV}$ were sent into the detector at an angle $\theta_{z}$ with respect to the fiber axis. For most of the results reported here, $\theta_{z}$ was $2^{\circ}$. The beam particle rates were $10^{2}-10^{3}$ events per spill $(2.6 \mathrm{~s})$. At high energies $(\geq 40 \mathrm{GeV})$ the beams were very clean, the contamination of electrons (pions) in the pion (electron) beam being below the $1 \%$ level. At lower energies this was different. Therefore, the event samples taken at these energies were cleaned up using data from a preshower counter (S6, see fig. 3) that was installed just upstream of the calorimeter. This preshower detector consisted of an absorber sheet $\left(1.14 X_{0}\right.$ tungsten $+0.53 X_{0}$ lead), followed by a scintillation counter. The signal in this scintillator provided separation between electron and pion events [6]. At 5 and $10 \mathrm{GeV}$, the preshower counter

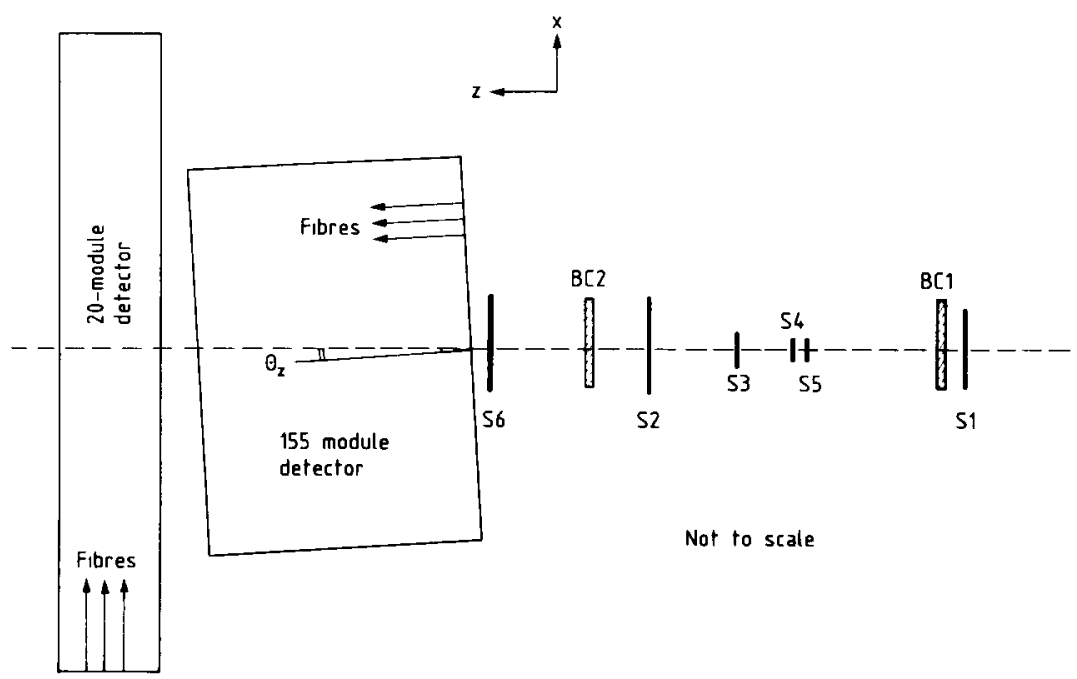

Fig. 3. Layout of the beam line, seen from above. In our convention, the angle $\theta_{z}$ shown in this figure has a positive sign. 
signal was part of the trigger. For electrons, the signal was required to be above the threshold, set at 7 times the minimum ionizing value, for pions the signal was required to be below this threshold. Since the preshower counter covered only an area of $15 \times 15 \mathrm{~cm}^{2}$ in the central region of the calorimeter, it was removed during the matrix scans (see sections 3.1, 4.1), where a larger area was investigated.

Off-line event selection required a single track, by cutting on the pulse height of the scintillation counters $\mathrm{S} 1, \mathrm{~S} 2$ and S3 between 0.5 and 1.7 times the minimum ionizing particle value. The $x$ and $y$ coordinates measured in the two beam chambers had to agree within 1 $\mathrm{cm}$. Beam halo particles were removed by cuts on $x$ and $y$ in the beam chambers.

\section{The position resolution for e.m. showers}

\subsection{Experimental data}

Many of the results described in this and the following sections were obtained from a matrix scan of the surface of the calorimeter with $80 \mathrm{GeV} \mathrm{e}^{-}$and $\pi^{-}$. A relatively broad beam was steered at $\theta_{z}=2^{\circ}$ into the detector. Data were taken at 49 different detector positions covering a grid of $12 \times 12 \mathrm{~cm}^{2}$ in the case of electrons. The impact points and the beam size are indicated in fig. 4. In this way, every part of the surface of a tower was hit by a sufficiently large number of electrons to allow for systematic studies of the position dependence of the results.

Such a systematic study was only done with the 80 $\mathrm{GeV}$ particle beams. The energy dependence of the results was studied with data taken at $\theta_{z}=3^{\circ}$ in the centre of one tower. The angular dependence was studied at $40 \mathrm{GeV}$, also for particles hitting the centre of a tower.

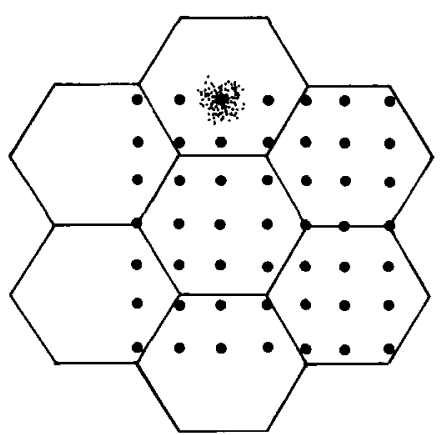

Fig. 4. The calorimeter area covered by the $80 \mathrm{GeV} \mathrm{e}^{-}$matrix scan. The dots correspond to the various beam positions; the size of the beam spot is indicated as well.
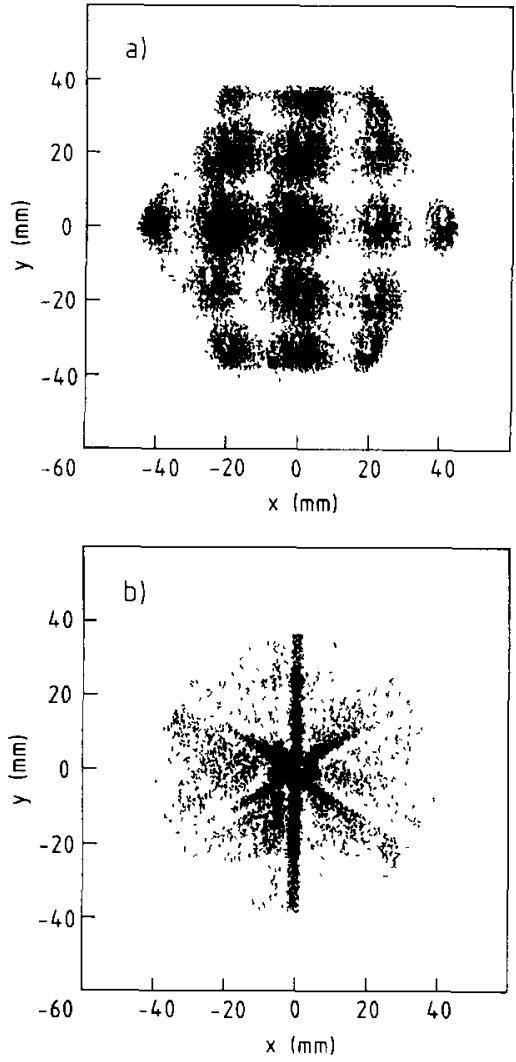

Fig. 5. Distribution of the impact point of $80 \mathrm{GeV}$ electrons over the surface of a calorimeter tower, as determined with the beam chambers (a) and from the calorimeter data with the simple centre of gravity method (b).

\subsection{Methods for determining the position resolution}

The standard way of determining the position of a particle showering in a calorimeter is by determining the centre of gravity $\bar{x}, \bar{y}$ of the energy $E$, deposited in the various detector cells (with coordinates $x_{1}, y_{l}$ ) that contribute to the signal,

$\bar{x}=\frac{\sum_{t} x_{t} E_{t}}{\sum_{t} E_{t}}$

and the same for the $\bar{y}$ coordinate. One tower of the calorimeter has an effective radius of $-1.6 R_{\mathbf{M}}$. As a consequence, an electron that hits the central region of a cell deposits a very large fraction of its energy in this one cell (typically 95\%). Most of the remaining $5 \%$ is shared between the six neighbouring towers. In practice, we did not consider towers located farther away from the shower axis, so that the centre of gravity was determined by summing over only seven towers. A displacement of the particle impact point by $\sim 1 \mathrm{~cm}$ from the tower centre only marginally affects the energy 


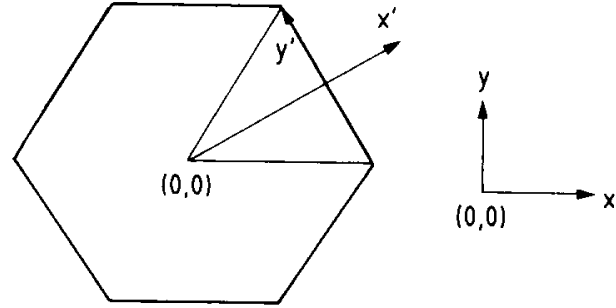

Fig. 6. The coordinate system used for determining the correct impact points from the energy deposit profiles. See text for detals.

sharing, so that the impact point is not correctly reconstructed in this case.

This is illustrated in fig. 5, which shows the impact point distribution measured with the beam chambers (fig. 5a) and the distribution of the impact points reconstructed from the centre of gravity of the measured energy deposit profiles (fig. 5b), for a sample of the recorded $80 \mathrm{GeV} \mathrm{e} e^{-}$events. The events tend to be shifted towards the centre of the tower when the calorimeter data are used in this way.
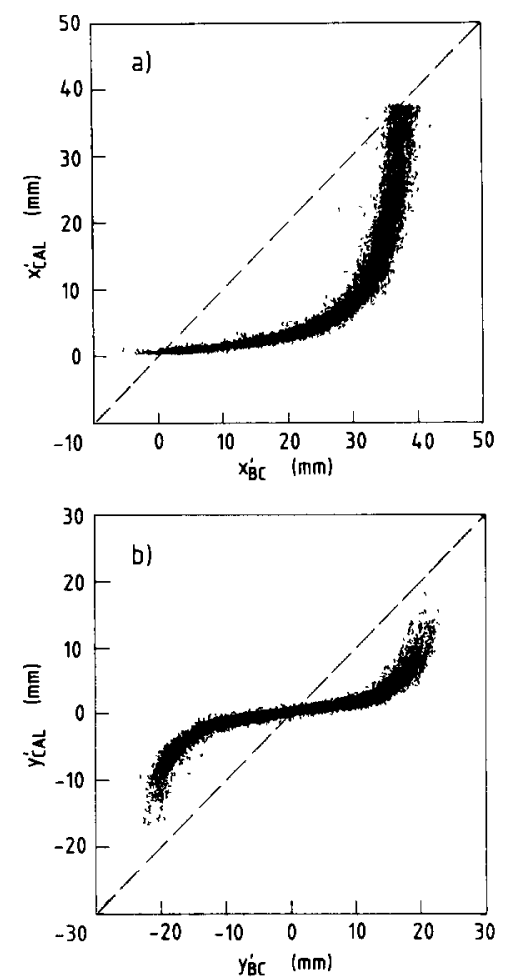

Fig. 7. Scatter plots for $80 \mathrm{GeV}$ electrons. The relation between the $x^{\prime}$ values measured with the beam chambers (horizontal) and the $x^{\prime}$ values determined from the calorimeter data with the centre of gravity method (vertical, a). The same for the $y^{\prime}$ values (b).
To study this effect more quantitatively, we defined a coordinate system adapted to the peculiarities of the hexagonal geometry in the following way (see fig. 6). An hexagon is composed of six equilateral triangles. The particles were attributed a position $\left(x^{\prime}, y^{\prime}\right)$, where the $y^{\prime}$ coordinate runs along the side of the hexagon that is part of the triangle in which the particle is located. The $x^{\prime}$ coordinate runs from the tower centre perpendicular to this side. We used the calorimeter data $(\bar{x}, \bar{y})$ to determine to which of the six triangles the event had to be attributed. The beam chamber $(x, y)$ values were subsequently calculated in the coordinate system $\left(x^{\prime}\right.$, $\left.y^{\prime}\right)$ defined by this particular triangle.

Fig. 7a shows the relation between the $x^{\prime}$ values of the impacting electrons, measured with the beam chambers (horizontal axis) and the $x^{\prime}$ values reconstructed from the energy deposit profiles (vertical axis). Ideally, all the points in this scatter plot should be located on the dashed line. Displacements of up to $2 \mathrm{~cm}$ are observed. Events are only reconstructed at approximately the right position if they occur near the tower
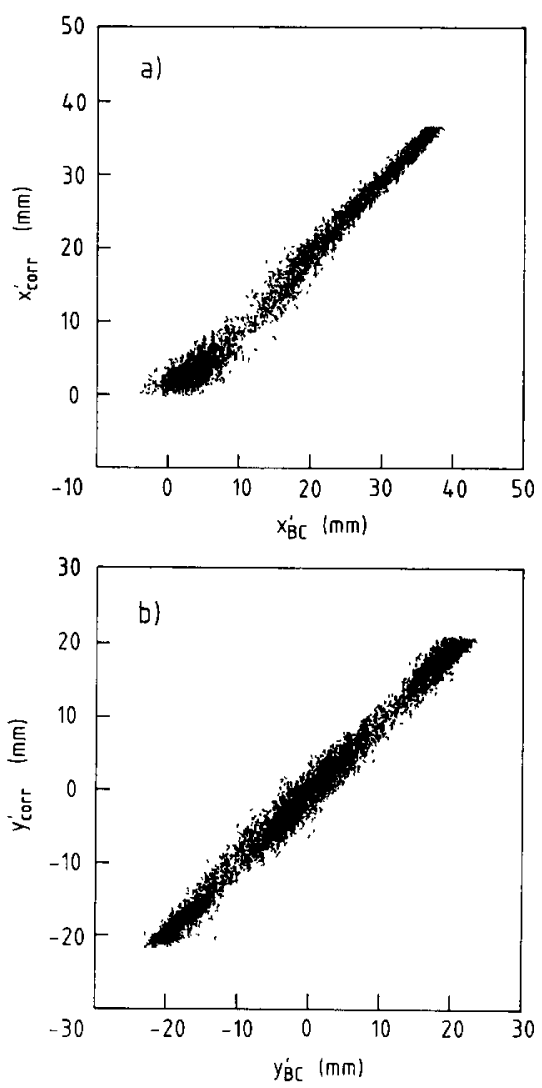

Fig. 8. Scatter plots for $80 \mathrm{GeV}$ electrons. The relation between the $x^{\prime}$ values measured with the beam chambers (horizontal) and the corrected $x^{\prime}$ values determined from the calorimeter information (vertical). See text for detalls (a). The same for the $y^{\prime}$ values (b). 
centre or near the boundary between towers. The same information is given for the $y^{\prime}$ coordinate in fig. $7 \mathrm{~b}$. Events only tend to be found at the correct position when they occur near the axis linking tower centres. In other regions displacements in $y^{\prime}$ of up to $1 \mathrm{~cm}$ are observed.

These phenomena are well known and several recipes for dealing with them have been proposed [7-9]. The methods proposed for curing the problem can be divided in two classes:

a) procedures that give an increased weight to towers with a small energy deposit;

b) procedures in which the centre of gravity found in the standard way is shifted, using an empirical algorithm.

We tried several methods and the best results were obtained with a procedure as in b). The reconstructed impact point was shifted to the approximately correct position using the following algorithm:

$x_{\text {corr }}^{\prime}=25.0 \arctan \left(0.363 x^{\prime}\right) \mathrm{mm}$,

$y_{\text {corr }}^{\prime}=14.7 \arctan \left(0.476 y^{\prime}\right) \mathrm{mm}$.

The relations between the $x^{\prime}\left(y^{\prime}\right)$ values measured with the beam chambers and the $x_{\text {corr }}^{\prime}\left(y_{\text {corr }}^{\prime}\right)$ values determined from the energy shower profiles and corrected in this way, are given in figs. $8 \mathrm{a}$ and $8 \mathrm{~b}$. The impact
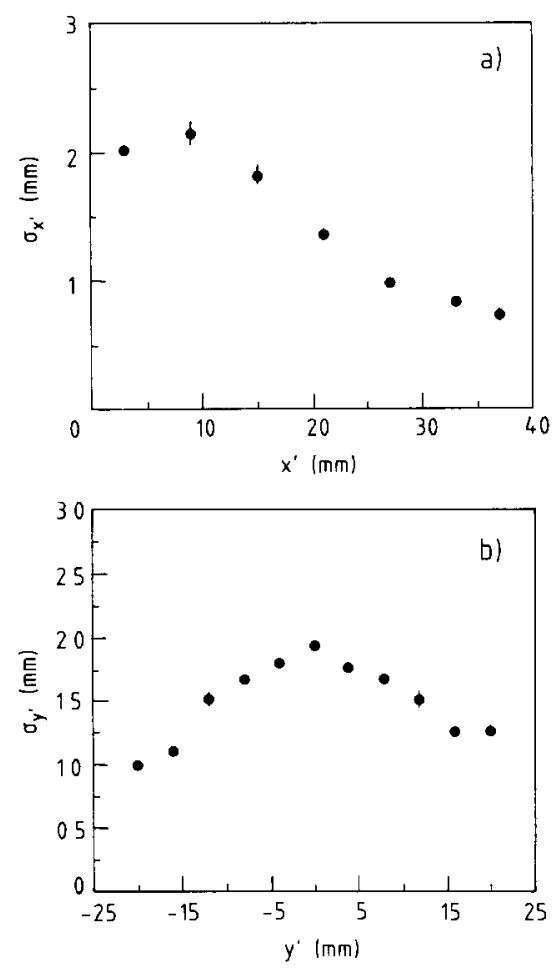

Fig. 9. The position resolution for $80 \mathrm{GeV}$ electrons as a function of $x^{\prime}$ and $y^{\prime}$.
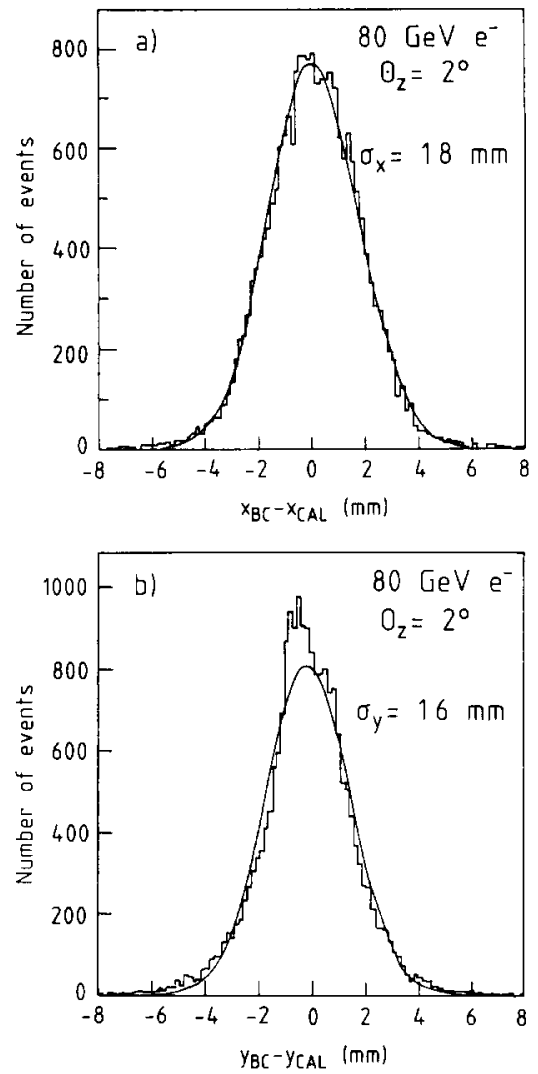

Fig. 10. Distribution of the differences between the $x$ coordinates measured with the beam chambers and those measured with the calorimeter, for $80 \mathrm{GeV} \mathrm{e}^{-}$uniformly distributed over a calorimeter tower (a). The same distribution for the $y$ coord1nates (b).

points are on average very well reproduced with this method.

\subsection{Experimental results}

The position resolution, given by the width of the bands in fig. 8 , is slightly dependent on the impact point of the electrons. Fig. 9 shows how the position resolution varies with $x^{\prime}$ and $y^{\prime}$. The best results were obtained in the corners where three modules join. On average, the RMS position resolutions $\sigma_{x}$ and $\sigma_{y}$ amount to 1.8 and $1.6 \mathrm{~mm}$ for $80 \mathrm{GeV}$ electrons (fig. 10).

The energy dependence of the position resolution was measured at $\theta_{z}=3^{\circ}$, for electrons at 5, 10, 20, 40, 80 and $150 \mathrm{GeV}$. Only data for particles in the central region, representing the worst case (see fig. 9) were available for this study. The data were treated in the same way as discussed before, using the same algorithm, eq. (2), for finding the correct impact points. In fig. 11, the position resolution for the central region of a tower is given as a function of the electron energy. The 


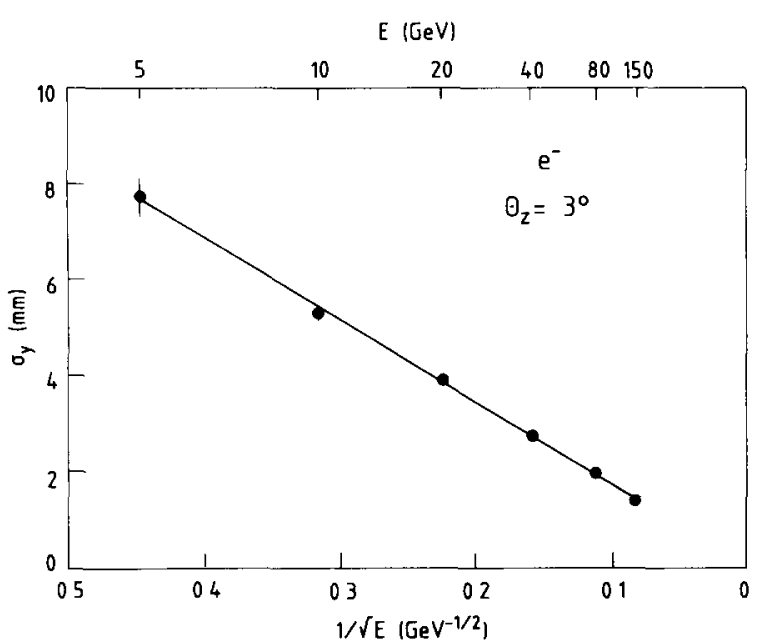

Fig. 11. The position resolution for electrons at $\theta_{z}=3^{\circ}$ as a function of the energy. Results for the central region of calorimeters towers. Averaged over the tower surface, the position resolution is $-20 \%$ smaller.

experimental data can be parametrized as $\sigma_{y}(E)=17.1 /$ $\sqrt{E}[\mathrm{~mm}]$, with $E$ in units of $\mathrm{GeV}$. For $\sigma_{x}$ the results were the same. From fig. 9, one may conclude that the average position resolution for electrons in this detector will be $-20 \%$ smaller than indicated in fig. 11 . Scaling with $1 / \sqrt{E}$ is expected, since the energy deposits $E_{1}$ in the cells $i$ (eq. (1)) have a relative accuracy $\sigma_{l} / \sqrt{E_{t}}$ which improves as $1 / \sqrt{E_{t}}$, if the shower profile stays the same.

The angular dependence of the position resolution was studied for $40 \mathrm{GeV}$ electrons entering the detector at angles $\theta_{z}$ varying between $0^{\circ}$ and $6^{\circ}$ with respect to the fiber axis. The results show essentially no effect of the angle on the position resolution (fig. 12). However,

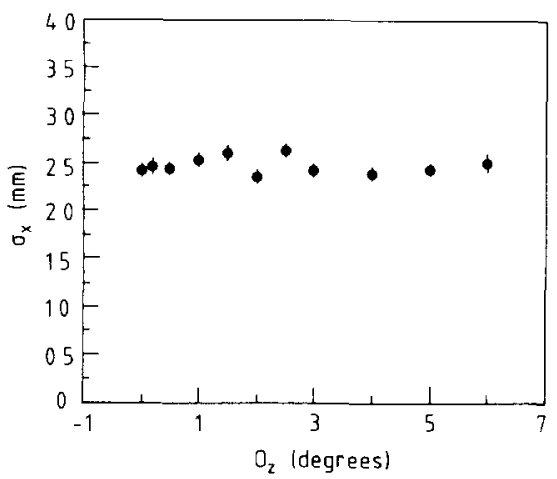

Fig. 12. The position resolution for $40 \mathrm{GeV} \mathrm{e} e^{-}$entering the detector in the centre of a tower, as a function of the angle $\theta_{z}$ between the particle direction and the fiber axis.
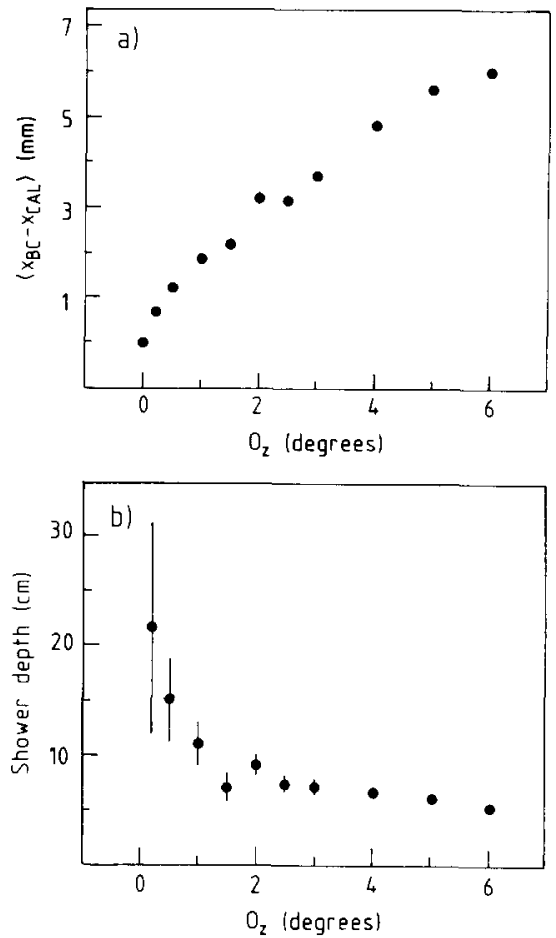

F1g. 13. The average position of the centre of gravity with respect to the impact point, as a function of the angle $\theta_{z}$ between the particle direction and the fiber axis (a). The average longitudinal centre of gravity as a function of $\theta_{z}$ (b). Data for $80 \mathrm{GeV}$ electrons.

we did observe another effect. In these measurements, the detector was always positioned in such a way that the beam hit the detector surface at the same spot, regardless of the angle $\theta_{z}$. Since the shower develops in depth, this will lead to a shift in the reconstructed centre of gravity with respect to the impact point. Fig. 13a shows this shift $(\Delta x)$ as a function of $\theta_{z}$. From this figure, one may determine the average longitudinal centre of gravity $\langle z\rangle$ of the showers, since $\langle z\rangle$ and $\Delta x$ are related as $\Delta x=\langle z\rangle \sin \left(\theta_{z}\right)$. Fig. $13 \mathrm{~b}$ shows $\langle z\rangle$ as a function of $\theta_{z}$. For angles larger than $1^{\circ}$, the average depth at which the light is produced by these $80 \mathrm{GeV}$ electrons showers is almost constant: $\langle z\rangle-6.5 \mathrm{~cm}$ $\left(\sim 9 X_{0}\right)$. For $\theta_{z}<1^{\circ}$ this depth increases, probably as a consequence of the fact that the incoming electrons may travel a considerable distance inside one fiber $\left(X_{0} \sim 40\right.$ $\mathrm{cm}$ for polystyrene), so that the shower develops deeper inside the detector [2].

This example shows how a good lateral position resolution translates into longitudinal sensitivity when the particles enter the detector at a nonzero angle with the fiber axis. This feature will be further exploited in section 6 . 


\section{The position resolution for hadronic showers}

\subsection{Experimental data}

The experimental data used for the determination of the hadronic position resolution were similar to the data described in the previous section:

- a $7 \times 7$ matrix scan with $80 \mathrm{GeV} \pi^{--}$, covering an area of $24 \times 24 \mathrm{~cm}^{2}$ in the central region of the detector, at $\theta_{z}=2^{\circ}$

- an angular scan with $40 \mathrm{GeV} \pi^{-}$in the centre of a tower;

- an energy scan at $\theta=3^{\circ}$ in the centre of a tower.

\subsection{Methods}

The method used to determine the hadronic position resolution was similar to the one described for e.m. showers. Although hadronic showers are much wider than e.m. ones, the displacement effects shown in fig. 7, which are caused by the fact that the lateral shower dimensions are small compared to the granularity, were also observed here, be it less pronounced.

This is illustrated in fig. 14, which shows the relation between the coordinates (again $x^{\prime}$ or $y^{\prime}$, see fig. 6) measured with the beam chambers and the coordinates determined from the centre of gravity of the energy deposit profiles. The displacement effects were also here cancelled with an arctan correction function, be it that the coefficients were chosen slightly different from those in eq. (2) in order to achieve optimal reconstruction of the shower position,

$x_{\text {corr }}^{\prime}=29.7 \arctan \left(0.084 x^{\prime}\right) \mathrm{mm}$,

$y_{\text {corr }}^{\prime}=17.0 \arctan \left(0.147 y^{\prime}\right) \mathrm{mm}$.

These displacement effects are probably due to the e.m. component in the shower, which accounts on average for about half of the energy deposit at $80 \mathrm{GeV}$.
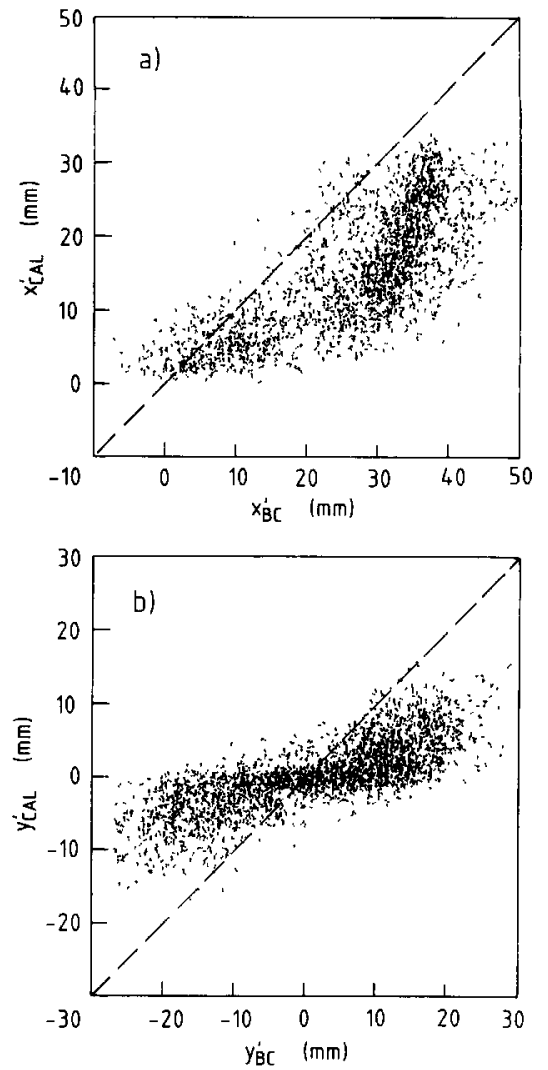

Fig. 14. Scatter plots for $80 \mathrm{GeV} \pi^{-}$. The relation between the $x^{\prime}$ values measured with the beam chambers (horizontal) and the $x^{\prime}$ values derived from the calorimeter data with the centre of gravity method (a). The same for the $y^{\prime}$ values (b).

Fig. 15 shows a typical hadronic event in the SPACAL calorimeter. The numbers correspond to the energy deposited in the individual towers. For this event, more than half of the energy was deposited in one

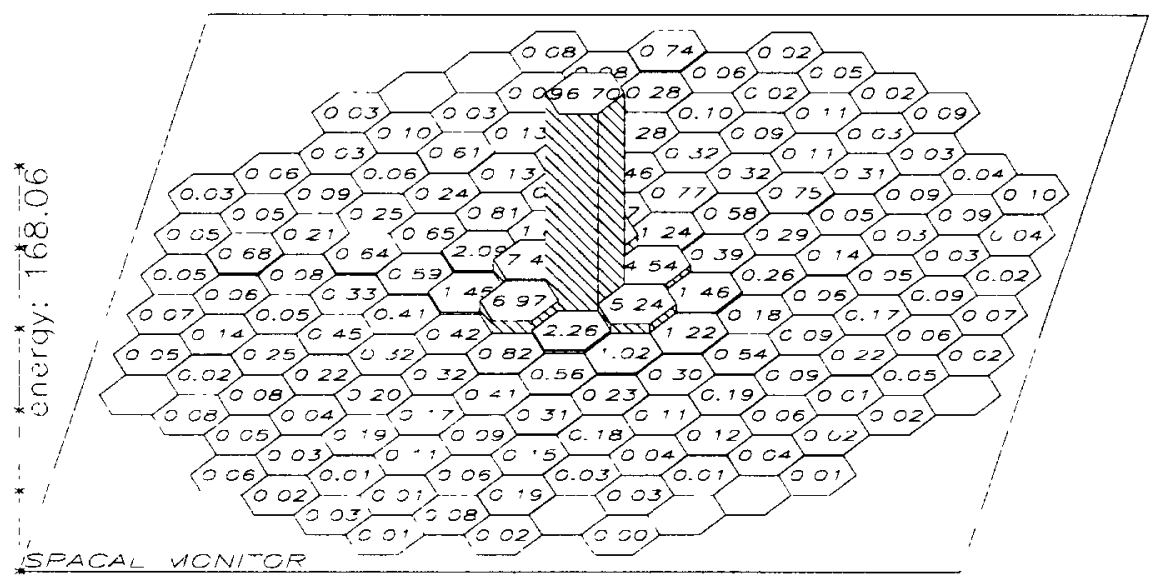

Fig. 15. Display of a typical hadronic event in SPACAL. The numbers correspond to the energy deposited in the individual towers. 
tower, the rest was distributed over essentially all the other towers. The question arises how far away from the shower axis should one go in order to calculate the centre of gravity, since many small energy deposits with large weight factors might deteriorate the accuracy of the position determination.

We studied the question of which towers should be used for the calculation of the centre of gravity in two ways:

a) The position resolution was determined as a function of the number of rings around the tower with the largest energy deposit that were taken into account - in other words, as a function of the radius of the cylinder that was assumed to contain the event for this purpose. The best resolution was found when two hexagonal rings around the shower axis were used (19 towers).

b) The position resolution was determined by considering only towers containing a certain minimum fraction of the total energy. Optimal resolution was found when only towers containing at least $5 \%$ of the total energy were used for calculating the centre of gravity. This method uses in practice a much smaller number of towers than 19 , required by the first method.

In general, the position resolution obtained with the first method was slightly better. Only for events in which there is not a single tower that absorbs a major fraction of the pion energy, the results are comparable. For such events, the position resolution also tends to be somewhat better than for the events of the type shown in fig. 15. This is illustrated in fig. 16, where the hadronic position resolution is given for both methods, as a function of the maximum energy fraction deposited in one single tower.

In practice, we used method a), with 19 towers, for determining the centre of gravity. The displacement

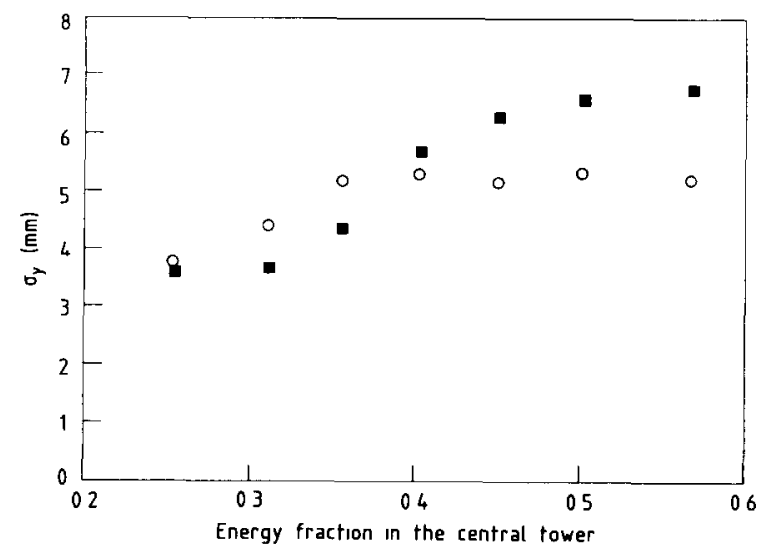

Fig. 16. The position resolution as a function of the maximum energy fraction deposited in one single tower. The open circles show the results obtained when 19 towers around the shower axis were used for calculating the centre of gravity, the squares consider only towers containing at least $5 \%$ of the total energy.
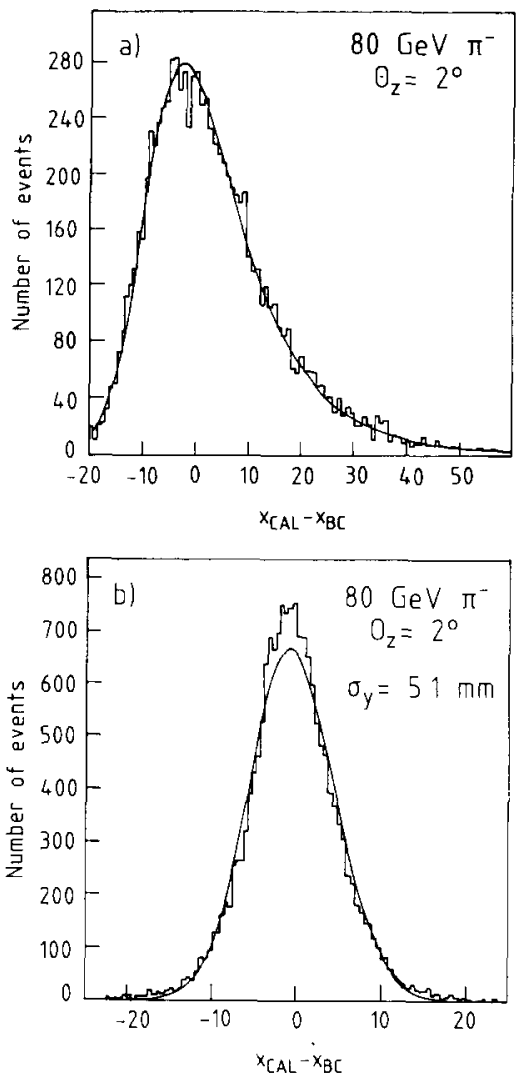

Fig. 17. Distribution of the differences between the impact point measured with the beam chambers and the centre of gravity of the $80 \mathrm{GeV} \pi^{-}$showers. Results for the $x$ (a) and $y$ (b) coordinates.

effects (fig. 14) were corrected for with the same procedure as for the e.m. showers (section 3.2).

\subsection{Experimental results}

A pion penetrating the calorimeter will undergo its first nuclear interaction at a depth $z$, of which the probability is distributed as $\exp \left(-z / \lambda_{\pi}\right)$. Also, depending on the type of interaction, the longitudinal development of the shower may differ strongly from event to event. Therefore, large variations occur in the longitudinal distribution of the light production in pion showers. If the beam enters the detector at an angle $\theta_{z}$ with respect to the fiber axis, these differences will translate into fluctuations of the reconstructed lateral centre of gravity which respect to the impact point.

The beam entered the detector in the $x-z$ plane at an angle $\theta_{z}$ with respect to the $z$-axis (see figs. 3 and 6 ). This means that the effect just mentioned should only be measurable in the position resolution for the $x$ coordinate, and not for the $y$ coordinate. Fig. 17 shows the distribution of the calculated centre of gravity with 


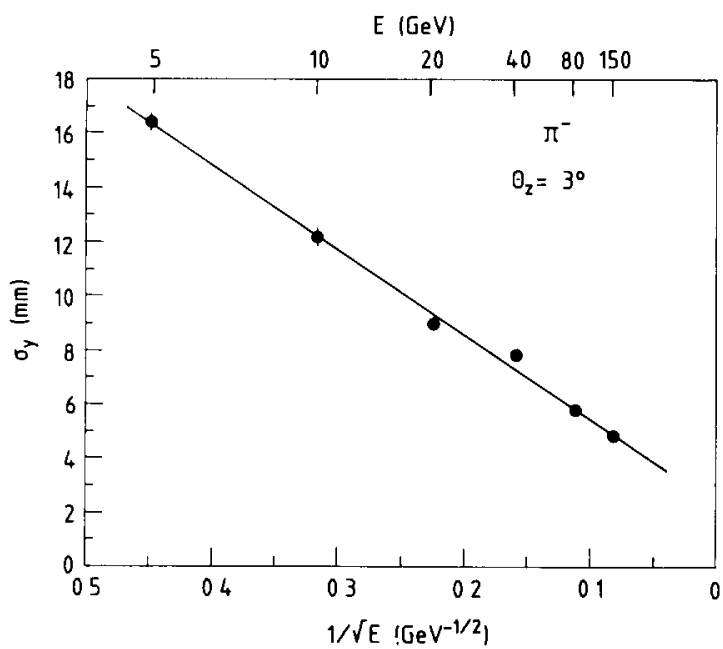

Fig. 18. The energy dependence of the hadronic position resolution, for pions entering the central region of a tower at $\theta_{z}=3^{\circ}$.

respect to the impact point for the $x$ coordinate (fig. 17a) and for $y$ (fig. 17b), for $80 \mathrm{GeV}$ pions at $\theta_{z}=2^{\circ}$, whose impact points were uniformly distributed over the tower surface. The $x$ distribution exhibits a clear exponential tail on the right hand side, reflecting the fluctuations in the starting points of the showers. The $x$ distribution is well described by a convolution of a Gaussian and an exponential distribution (fig. 17a) in which the width of the Gaussian part is (almost) equal to the one for the $y$ distribution, and the slope of the exponential part equals $11 \mathrm{~mm}$. From this one can infer the interaction length for pions $\lambda_{\pi}$ in this detector as $11 / \sin \left(2^{\circ}\right)=315 \mathrm{~mm}$. This measurement therefore confirms that the interaction length for pions is $50 \%$ larger than for protons [5].

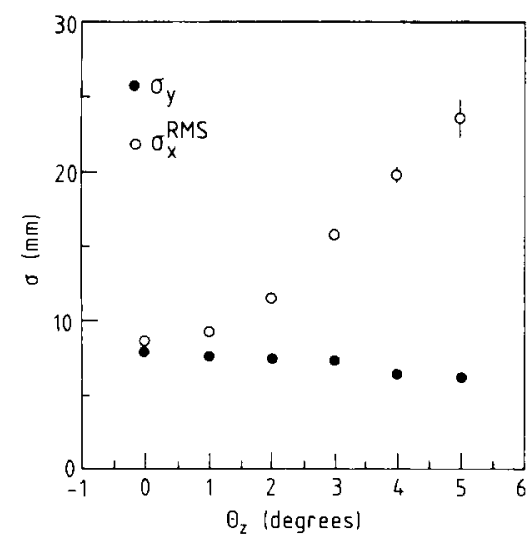

Fig. 19. The angular dependence of the hadronic position resolution, for $40 \mathrm{GeV} \pi^{-}$entering the central region of a tower.

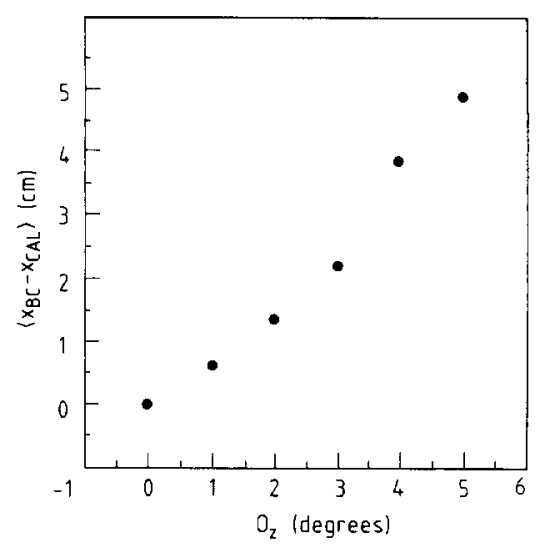

Fig. 20. The average shift of the centre of gravity in the $x$ direction, with respect to the impact point measured with the beam chambers, as a function of $\theta_{z}$. Data for $40 \mathrm{GeV} \pi^{-}$ entering the central region of a tower.

The position resolution for pions depends on the impact point, in the same way as for electrons (see fig. 9), be it that the effects are less pronounced. At $80 \mathrm{GeV}$, the average value of $\sigma_{y}$ amounts to $5.1 \mathrm{~mm}$, whereas in the center of a tower $\sigma_{y}=5.8 \mathrm{~mm}$ was found. In fig. 18, the energy dependence of the hadronic position resolution is given for pions entering the central region of a tower at $\theta_{z}=3^{\circ}$. The experimental data can be parametrized as $\sigma_{y}(E)=2.4+31.4 / \sqrt{E}$ [mm], with $E$ in $\mathrm{GeV}$. The constant term may be caused by the large nonGaussian event-to-event fluctuations in the magnitude of the e.m. component of the showers.

The angular dependence is shown in fig. 19. The resolution in $y$ is more or less independent of $\theta_{z}$, but the exponential tail on the right hand side of the $x$ distribution becomes of course more and more pronounced at increasing angles, causing $\sigma_{x}$ to increase. Fig. 20 shows the average shift of the centre of gravity as a function of $\theta_{z}$. As for e.m. showers (fig. 13), this effect allows one to determine the average depth at which the energy is deposited. We found that this occurs at $z \sim 55 \mathrm{~cm}\left(\sim 2.5 \lambda_{\mathrm{I}}\right)$.

\section{Particle-particle separation}

One tends to think of hadronic showers as phenomena that require quite a large detector volume to be detected in. This is certainly true if one wants to (almost) contain the showers or if one wants to do an accurate measurement of the energy of the showering particle. The SPACAL detector was designed for this purpose and needed an instrumented mass of more than 13 tons to achieve this goal.

However, when it comes to localizing the showering particle, the situation is quite different. Fig. 15 shows 
that in a typical hadronic event at $80 \mathrm{GeV}$ more than half of the shower energy is deposited in one tower, and the other half is distributed over the rest of the detector. This means that two particles hitting the detector and developing showers in it might be recognized as two separate particles even if they enter the detector at a relatively small distance from each other. The short radiation and interaction length of the detector are a favourable feature in this respect.

In this paper, we report only about the $\pi / \pi$ separation capability of this detector. The electron-pion separation, very relevant for the identification of electrons (e.g. from heavy flavour decay) inside jets, will be dealt with in a separate paper. We note that, because the electron showers are narrow, they can be better identified at a given distance from a showering pion and, therefore, the $e / \pi$ separation works well down to shorter distances than for $\pi / \pi$.

The $\pi / \pi$ separation was investigated in the following way. We merged the showers from two pions hitting the detector at a relative distance $d$. The resulting energy deposit profile was compared to the average shower profile for one pion hitting the detector at an intermediate position. We mainly used $80 \mathrm{GeV} \pi^{-}$data from the matrix scan for this purpose, since this data allowed for a systematic study of the results as a function of $d$.

We found that the fractional energy deposit, $f(r)$, in a given cell whose centre is located at a distance $r$ from the centre of gravity of the shower, could be fairly accurately parametrized with the sum of a Gaussian and an exponential function,

$f(r)=C \exp \left(-a r^{2}\right)+D \exp (-b r)$,

with $a=0.070 \mathrm{~cm}^{-2}, b=0.230 \mathrm{~cm}^{-1}, C=0.548$ and $C=0.192$, for the region $0<r<17 \mathrm{~cm}$. It should be emphasized that $f(r)$ is not the lateral shower profile, since the effects of the finite cell size, which are very important close to the shower axis, were not unfolded.

Next, a criterion $K$ was defined in order to study how well individual showers are described by $f(r)$. Good results were obtained with a criterion of the following type:

$K=\sum_{l} r_{t}\left[E_{l}-f\left(r_{t}\right)\right]^{2}$,

where $E_{1}$ is the fraction of the total energy that is deposited in tower $i$ and $r_{t}$ the distance from the centre of tower $i$ to the (uncorrected) centre of gravity of the energy deposit profile, as calculated with eq. (1). In practice, only the towers in the first two hexagonal rings surrounding the tower containing the centre of gravity were used for calculating $K$. Including more distant towers did not improve the results.

In fig. $21, K$ distributions are given for single pions and for $80+80 \mathrm{GeV}$ pions at a relative distance $d$ of 9
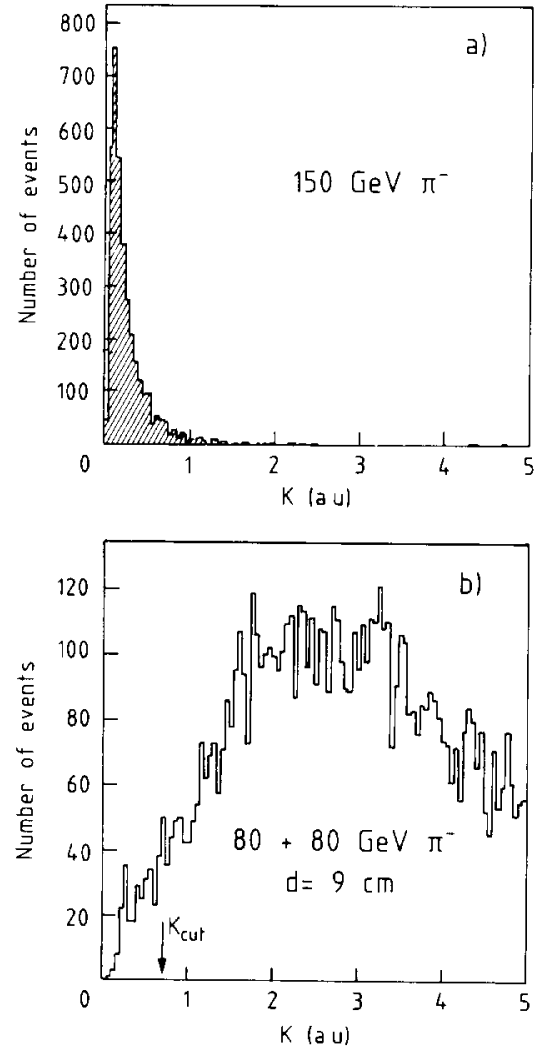

Fig. 21. Distribution of the $\pi / \pi$ separation criterion $K$ for single $\pi^{-}$showers at $150 \mathrm{GeV}$ (a), and for combinations of two $80 \mathrm{GeV}$ pions hitting the detector $9 \mathrm{~cm}$ apart (b).

$\mathrm{cm}$. The width of the distribution for the single pions is a measure of the lateral shower-to-shower fluctuations at this energy. The $K$-distribution for the $80+80 \mathrm{GeV}$

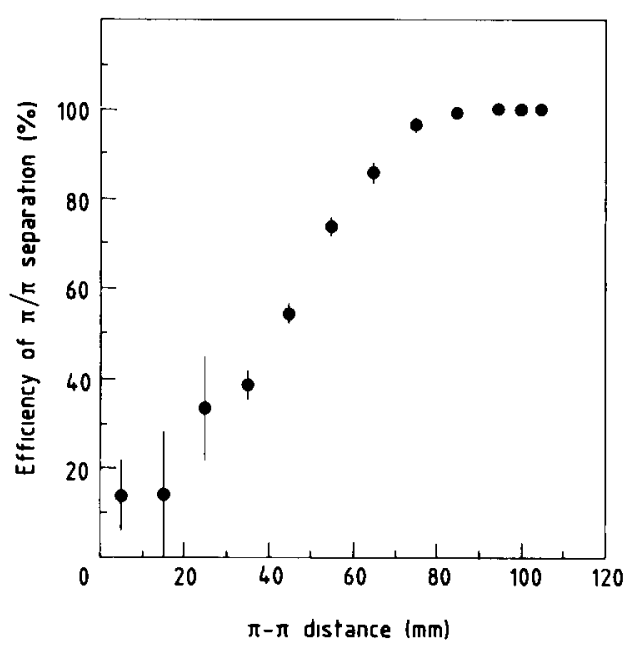

Fig. 22. The probability of recognizing a $80+80 \mathrm{GeV} \pi^{-}$pair as a function of their relative distance $d$, using a criterion that recognized single pions in $95 \%$ of the cases as such. 


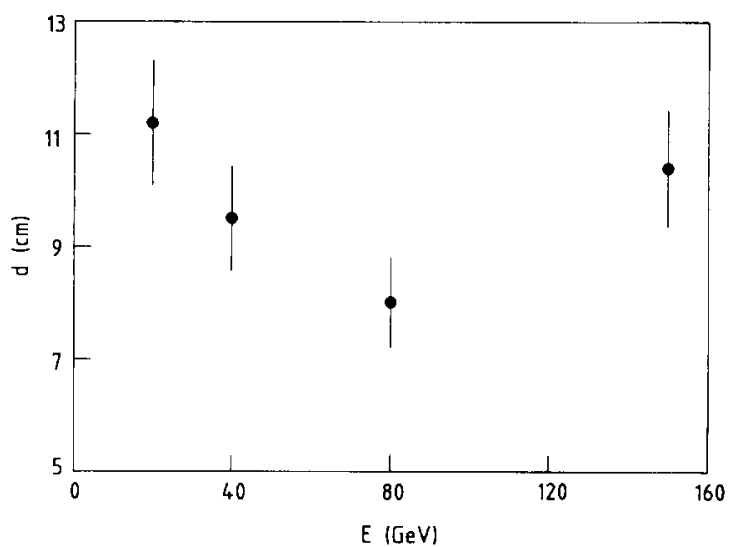

Fig. 23. The distance $d$ at which $95 \% \pi / \pi$ separation is achieved, as a function of the pion energy $E$ (for $80+E \mathrm{GeV}$ pion pairs).

$\pi^{-}$combinations peaks at much higher values, indicating that the energy deposit profile of these events is in general poorly described by the typical one for a single pion at approximately twice the energy.

In order to identify pion pairs, a cut on $K$ was applied. All the events with $K<K_{\text {cut }}$ were considered to be single pions, all the other events were considered to be pion pairs. We chose $K_{\text {cut }}$ in such a way that $95 \%$ of the single pions came out as single pions, and determined the probability of recognizing a pion pair as a function of $d$. The result is given in fig. 22. One sees that the $\pi / \pi$ separation is better than $95 \%$ for $d \geq 8 \mathrm{~cm}$.

The energy dependence of the $\pi / \pi$ separation capability was studied by merging pions of $E \mathrm{GeV}$ hitting the centre of the detector and $80 \mathrm{GeV}$ pions entering at a distance $d$ from the centre. As a reference profile, pion data at 80 and $150 \mathrm{GeV}$ were used. Since the average pion profiles at these energies are not very different, the results were almost independent of the reference profile used. In fig. 23 the results of this analysis are given. The distance at which $95 \% \pi / \pi$ separation is achieved $\left(d_{0.95}\right)$ is shown as a function of the energy. The error bars represent the fluctuations that were observed when pions at a distance $d$ from the centre were merged with different pions of $E \mathrm{GeV}$ (hitting the centre). As can be expected, the separation works best for two particles of equal energy.

From the matrix scan data, it was found that the $K$ distribution for single pions at $80 \mathrm{GeV}$ was almost independent of the impact point of the pions. Also, the $\pi / \pi$ separation capability $d_{0.95}$ and the variations in it were not significantly different from the values shown in fig. 23 when $80 \mathrm{GeV}$ pions were merged with $80 \mathrm{GeV}$ pions entering at positions other than the centre of the detector. This indicates that the $\pi / \pi$ separation is relatively insensitive to the impact point of the particles.

These results are encouraging, since they open a window to analysis of jet structures in $4 \pi$ experiments at colliders. Fig. 24 displays a multiparticle event, obtained with an interaction trigger. The pions were required to interact in an upstream target and the reaction products were detected with the calorimeter. Several particles produced in this interaction can be clearly recognized from the hit pattern in the calorimeter.

\section{Applications}

In sections 3 and 4 we have already shown how the good lateral position resolution may yield information on the longitudinal shower development, when the particles enter the detector under a small angle with the fiber axis. In particular, it was possible to determine the

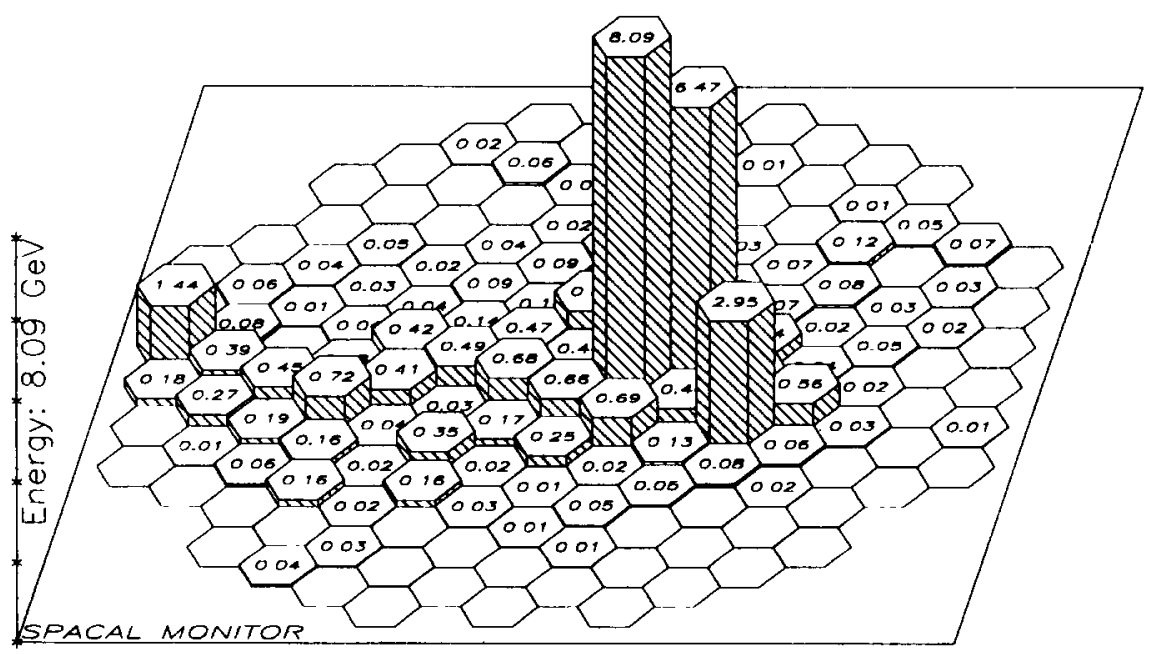

Fig. 24. Event display of a pion interacting in an upstream target, the reaction products being detected by the calorimeter. 
average depth at which the scintillation light was produced.

In this section, we will show how this information may be used on an event-by-event basis, and how it then may improve the detector performance. Two examples will be given of this.

\subsection{Improvement of the hadronic energy resolution}

The first example concerns the hadronic signal distribution. Hadronic showers exhibit large fluctuations in their longitudinal energy deposit profile (see section 4.3). In addition, the response of the scintillating fibers is not uniform in depth, because of light attenuation. Therefore, light attenuation in the fibers will lead to a systematic, instrumental effect contributing to the energy resolution for hadronic shower detection [10]. This effect is in first approximation independent of the
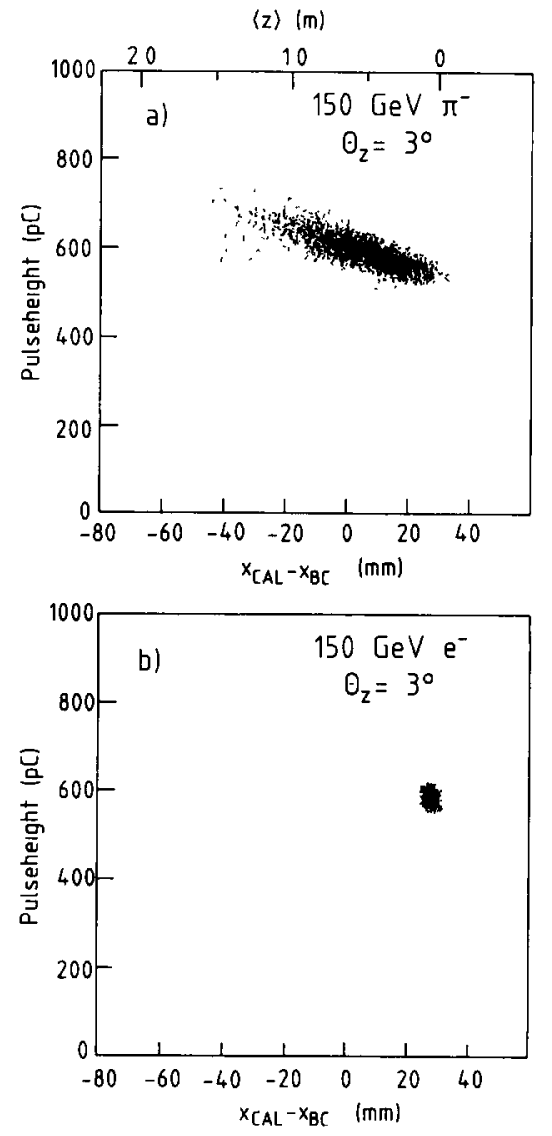

Fig. 25. Scatter plot showing the calorimeter signal (vertical) versus the displacement of the shower centre of gravity with respect to the particle's impact point (horizontal). Data for 150 $\mathrm{GeV} \pi^{-}$(a) and $150 \mathrm{GeV} \mathrm{e}^{-}$(b), at $\theta_{z}=3^{\circ}$. The effective depth $\langle z\rangle$ of the light production (see text) is shown on the top horizontal axis. The coordinates $x_{\mathrm{CAL}}-x_{\mathrm{BC}}$ have an offset (see ref. [11]).
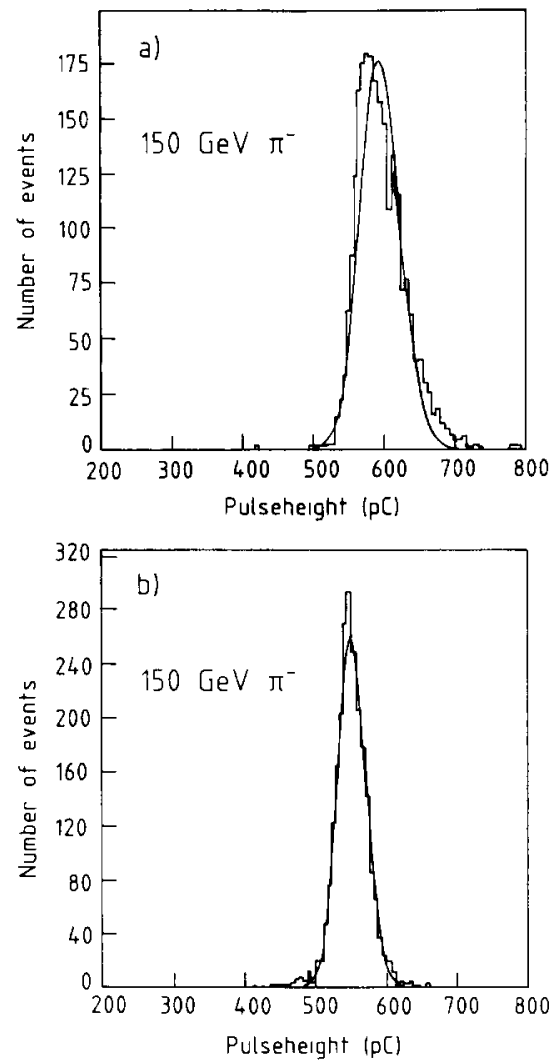

Fig. 26. The signal distribution for $150 \mathrm{GeV} \pi^{-}$before (a) and after (b) correcting for the effects of light attenuation in the fibers. The smooth curves are the results of Gaussian fits to the data.

hadron energy and will therefore give rise to a constant term in the energy resolution.

In the development of this detector, much emphasis was given to improving the light attenuation characteristics of the fibers [2]. Nevertheless, the remaining effects are still significant, particularly at very high energies, where the effect of a constant term in the energy resolution is of course relatively largest.

Knowledge of the average depth of the light production event-by-event makes it possible to considerably reduce the effects of light attenuation and, therefore, improves the hadronic energy resolution. This is illustrated with $150 \mathrm{GeV} \pi^{-}$data, taken at $\theta_{z}=3^{\circ}$.

In fig. 25a, the signal distribution for individual events is shown versus the displacement of the centre of gravity with respect to the impact point of the pions. For comparison, the same plot is given for electrons (fig. 25b). The displacement $\Delta x\left(=x_{\mathrm{CAL}}-x_{\mathrm{BC}}\right)$ and the average depth $\langle z\rangle$ at which the light is produced are related as $\Delta x=\langle z\rangle \sin \left(3^{\circ}\right)[11]$. And since the position resolution in the lateral plane amounts to $-4 \mathrm{~mm}$, the accuracy of the $\langle z\rangle$ values given in this plot amounts to 
about $8 \mathrm{~cm}$. Therefore, fig. $25 \mathrm{a}$ is a fairly accurate representation of the distribution of the longitudinal centres of gravity for the $150 \mathrm{GeV} \pi^{-}$showers.

Fig. 25 illustrates the difference in longitudinal shower development between e.m. and hadronic showers. The effect of light attenuation is clearly visible in that the average pion signal rises at increasing depth.

The energy resolution, obtained by projecting the distribution on the vertical axis, is therefore much worse than the width of the band in fig. 25a, which is determined by the fluctuations in the shower development itself. Moreover, the signal distribution is non-Gaussian as a consequence of the non-Gaussian event distribution in depth (see fig. 26a).

Using the attenuation curve of the fibers and the effective depth of the light production for each event, one can very simply eliminate this effect. This is shown in fig. 26b. To obtain this figure, the signals were weighted with a factor corresponding to an attenuation length of $7 \mathrm{~m}$. As a result of this procedure, the energy resolution $\sigma / E$ improved from $4.8 \%$ to $3.7 \%$ and the signal shape became almost Gaussian.

It should be emphasized that this example concerns the detection of single pions. The applicability of this method for jets is very questionable. However, jet detection is much less sensitive to the effects of light attenuation in the fibers, since a jet consists of many particles that develop showers simultaneously, and therefore the fluctuations in the longitudinal light production profile are much less important [10]. In this respect, a jet may be considered a hadronic shower with a fixed starting point, i.e. the interaction vertex.

\subsection{Improvement of the $e / \pi$ separation}

Electron identification is a major task of calorimeters in modern experiments. The Spaghetti Calorimeter offers excellent possibilities in this respect. We have developed several methods that allow $e / \pi$ separation at the $10^{-3}-10^{-4}$ level, including one that might be applied at the first trigger level [6].

Usually, the methods exploit the fine lateral granularity of the detector, which makes it possible to distinguish electrons and pions through the differences in the lateral shower development. Fig. 27 shows an example of the quality of the separation that can be achieved in this way. We defined a quantity $R_{p}$ as

$R_{\mathrm{p}}=\frac{\sum_{t} r_{t} E_{t}^{04}}{\sum_{t} E_{l}^{04}}$,

where $E_{1}$ is the energy deposited in an individual tower $i$ and $r_{1}$ is the distance between the centre of tower $i$ and the (uncorrected) centre of gravity of the energy deposit profile as in eq. (1). All 155 towers were used

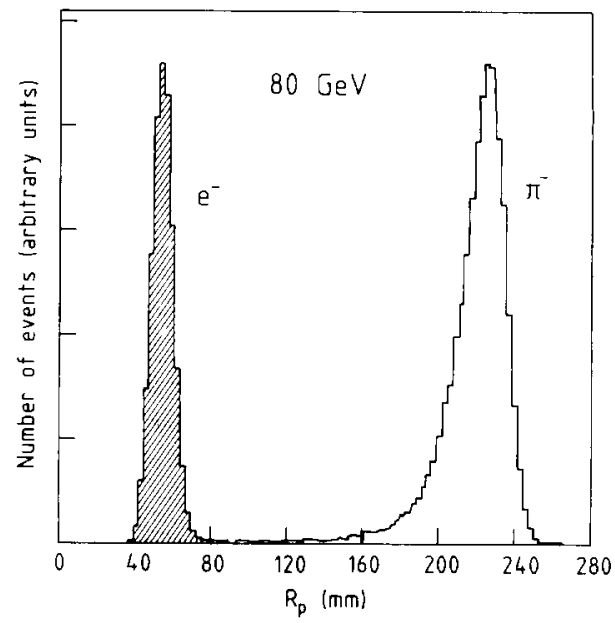

Fig. 27. Distribution of the effective width $R_{\mathrm{p}}$ (see text) for electron and pion showers at $80 \mathrm{GeV}$ and $\theta_{z}=2^{\circ}$.

for calculating $R_{\mathrm{p}}$. Electron and pion showers are very cleanly separated using this parameter, which is a measure of the effective shower width.

However, a small fraction (order $10^{-3}$ ) of the pion events cannot be separated from electrons with such a criterion. These are presumably events where (almost) all the pion energy is transferred to $\pi^{0}$ 's in the first interaction, and therefore the showers look laterally very much like e.m. ones.

The starting point of such showers occurs at a certain depth $z$ inside the calorimeter, with a probability

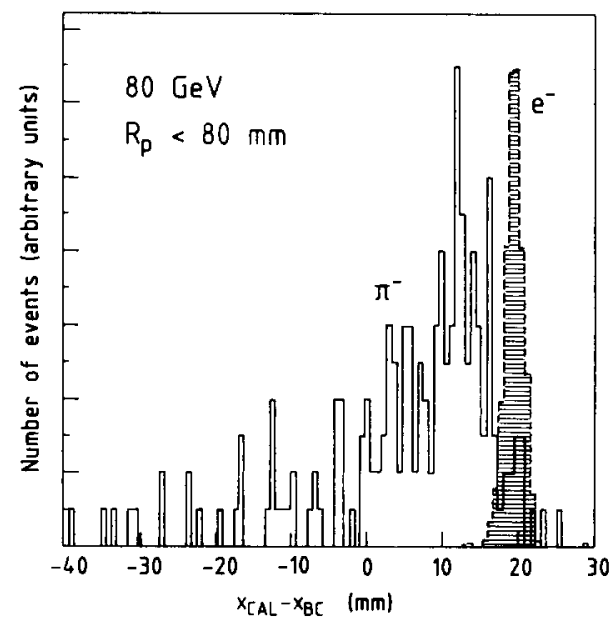

Fig. 28. Distribution of the displacement of the shower centre of gravity with respect to the particle impact point for electrons and for pions that produce showers that are laterally indistinguishable from electrons. Data for $80 \mathrm{GeV}$ particles at $\theta_{z}=2^{\circ}$. The coordinates $x_{\mathrm{CAL}}-x_{\mathrm{BC}}$ have an offset (see ref. [11]). 
distributed as $\exp \left(-z / \lambda_{\pi}\right)$, so that one may distinguish them from genuine electron showers using the shift between the lateral centre of gravity and the impact point (cf. figs. 25a and 25b). This works very well.

We selected the pion events from fig. 27 that could not be distinguished on the basis of their lateral shower profile, i.e. at $R_{\mathrm{p}}<80 \mathrm{~mm}$. The distribution of their centre of gravity displacement with respect to the pion impact point is given in fig. 28. For comparison, the distribution for electrons is given as well. Thanks to the good position resolution, all the pions interacting beyond a few centimetres in depth can be distinguished from electrons in this way. The final $e / \pi$ separation achieved by combining both the lateral and longitudinal shower characteristics amounted to $1.5 \times 10^{-4}$ at 80 $\mathrm{GeV}$, for $98 \%$ electron efficiency.

\section{Summary and conclusions}

We have studied the performance of a full-size lead/scintillating-fiber calorimeter, and in particular its capability of localizing the particles that develop showers in it. The calorimeter consisted of longitudinally unsegmented hexagonal towers with an effective radius of 39 $\mathrm{mm}\left(=1.6 R_{\mathrm{M}}\right.$ or $\left.0.19 \lambda_{\mathrm{I}}\right)$.

For e.m. showers we found that the position resolution significantly depends on the region of a tower where the particle entered. The best resolutions were found in the regions where three modules join, $\sigma \sim 1.0$ $\mathrm{mm}$ at $80 \mathrm{GeV}$. In the central region a resolution of $\sim 2$ mm was obtained at this energy. Averaged over the tower surface we measured $1.7 \mathrm{~mm}$. For other energies, we found that these numbers scale as $1 / \sqrt{E}$. The position resolution does not depend on the angle $\theta_{z}$ between the particle's trajectory and the fiber axis, for $\theta_{z}<6^{\circ}$.

For hadronic showers, the position resolution is less sensitive to the impact point. At $80 \mathrm{GeV}$, we obtained $5.1 \mathrm{~mm}$ accuracy. Also here, the $1 / \sqrt{E}$ scaling law applies, be it with an offset of $-2 \mathrm{~mm}$. When the detector is tilted at a small angle, the position resolution in the coordinate that runs in the tilted plane deteriorates because of the fluctuations in the starting point of the shower. We observed a $50 \%$ increase for 40 $\mathrm{GeV}$ pions at $\theta_{z}=2^{\circ}$. The resolution in the coordinate orthogonal to the tilted plane is unaffected.

Due to the very high effective density of the detector, the lateral shower dimensions are small. Because of this and because of the fine detector granularity, we were able to separate two particles hitting the detector at relatively close positions in space by analyzing the energy deposit pattern. Two $80 \mathrm{GeV}$ pions could be separated in more than $95 \%$ of the cases down to a distance of $8 \mathrm{~cm}$. This distance increased to $11 \mathrm{~cm}$ for a $20 \mathrm{GeV} / 80 \mathrm{GeV}$ pion pair.
In order to avoid the effects of anomalous sampling of e.m. showers in the early stage [2], this detector has to be placed in such a way that the particles to be detected enter it at a small angle (a few degrees) with respect to the fiber axis. This has turned out to have major beneficial effects for the localization of particles, since the good lateral position resolution translates in this way into sensitivity to details of the longitudinal shower development. Hence, a $2 \mathrm{~mm}$ lateral position resolution at $\theta_{z}=3^{\circ}$ gives $\sigma_{z} \sim 4 \mathrm{~cm}$. We demonstrated that this feature could be used to eliminate the effects of light attenuation on the energy resolution for single-pion detection and to improve the $\mathrm{e} / \pi$ separation capability considerably.

The SPACAL calorimeter was designed as an integrated detector for both e.m. and hadronic particle showers. Its lateral granularity, which in principle can be arbitrarily fine, was chosen as a compromise between what was believed to be needed for e.m. showers (fine granularity) and hadronic showers (crude granularity sufficient). In projective structures, needed for $4 \pi$ experiments at colliders, these different requirements would be automatically better matched. Our analysis shows, however, that a fine granularity is also very beneficial for hadronic shower detection. The fact that the position of the centre of gravity of the shower with respect to the impact point of the particle depends on the region of the tower where the particle entered, which is a typical sign of a granularity that is too crude, was even observed for hadronic showers (fig. 14). Therefore, all the results described in this paper would further improve with finer granularity.

\section{Acknowledgements}

We are very much indebted to the LAA project leader, Prof. A. Zichichi. We are grateful to P. Jenni who helped us in many ways, in particular when running the beam tests. The work described in this paper would have been impossible without the outstanding technical support provided by our technicians. $O$. Barnaba, M. Boriello, S. Bricola, J.-M. Chapuis, C. Farella, A. Freddi, G. Improta, G. Iuvino, F. Pagano, R. Rocco, L. Rose-Dulcina, G. Sannier, A. Sigrist and V. Vanzanella, Financial support from the Istituto $\mathrm{Na}$ zionale di Fisica Nucleare to the Cagliari, Napoli and Pavia groups, from the Conselho Nacional de Desenvolvimento Cientifico e Technologico of Brazil to the Rio de Janeiro group, and from the Department of Energy to the San Diego group is acknowledged. And finally, we are grateful to the staff of the SPS, and in particular to N. Dobble, for the excellent beam conditions provided during our tests. 


\section{References}

[1] R. DeSalvo et al., Nucl. Instr. and Meth. A279 (1989) 467.

[2] D. Acosta et al., Nucl. Instr. and Meth. A294 (1990) 193.

[3] R. Wigmans, Nucl. Instr. and Meth. A259 (1987) 389.

[4] E. Bernardi et al., Nucl. Instr. and Meth. A262 (1987) 229.

[5] We define the nuclear interaction length $\lambda_{1}$ in the same way as the Particle Data Group in Phys. Lett. B239 (1990), page III 5, 6. It is the mean free path for protons between inelastic interactions. We will use the symbol $\lambda_{\pi}$ for pions. Since the inelastic cross sections for protons are 50\% larger than for pions, $\lambda_{\pi} \approx 1.5 \lambda_{1}$.

[6] D. Acosta et al., Nucl. Instr. and Meth. A302 (1991) 36.

[7] G.A. Akopdjanov et al., Nucl. Instr. and Meth. 140 (1977) 441.

[8] R.L. Carrington et al., Nucl. Instr. and Meth. 163 (1979) 203.

[9] S. Orito and T. Kobayashi, Nucl. Instr. and Meth. 215 (1983) 93.
[10] F.G. Hartjes and R. Wigmans, Nucl. Instr. and Meth. A277 (1989) 379.

[11] In figs. 25 and 28 , the horizontal coordinates $x_{\mathrm{CAL}}-x_{\mathrm{BC}}$ are offset by a few $\mathrm{cm}$ because of the following reason. When the detector was operated at a certain angle $\theta_{z}$, it was displaced over a certain distance in the $x$ direction such as to achieve that the light production would be maximum at the position aimed at (usually the centre of the detector). At $\theta_{z}=3^{\circ}$ (fig. 25), the electrons and pions crossed the detector surface at a relative distance of 36 $\mathrm{mm}$ to achieve this goal, at $\theta_{z}=2^{\circ}$ (fig. 28) it was $24 \mathrm{~mm}$. In order to compare the $\Delta x$ distributions for electrons and pions, the $x_{\mathrm{CAL}}$ values for electrons were increased by the appropriate amount. The offset in the $x_{\mathrm{CAL}}-x_{\mathrm{BC}}$ values is in this way equalized for electrons and pions. Other contributions to the offset come from the effects shown in figs. 13a (electrons) and 20 (pions). The offset has been eliminated in the top axis of fig. 25 . 